\title{
Effects of paroxetine-mediated inhibition of GRK2 expression on depression and cardiovascular function in patients with myocardial infarction
}

This article was published in the following Dove Press journal:

Neuropsychiatric Disease and Treatment

13 September 2016

Number of times this article has been viewed

\author{
Xiuqing Tian' \\ Qing Wang' \\ Rui Guo ${ }^{2}$ \\ Lingling $\mathrm{Xu}^{3}$ \\ Qin M Chen ${ }^{4}$ \\ Yinglong Hou' \\ 'Department of Cardiology, \\ Qianfoshan Hospital of Shandong \\ University, Jinan City, ${ }^{2}$ Department \\ of Physiology, Nanjing University \\ of Chinese Medicine, Nanjing City, \\ ${ }^{3}$ College of Medicine, Weifang Medical \\ University, Weifang City, People's \\ Republic of China; ${ }^{4}$ Department of \\ Pharmacology, College of Medicine, \\ University of Arizona, Tucson, AZ, USA
}

Correspondence: Yinglong Hou

Department of Cardiology, Qianfoshan

Hospital of Shandong University,

16766 Jingshi Road, 2500I4, Jinan City,

People's Republic of China

Email hylong17@sina.com

\section{Qin M Chen}

Department of Pharmacology, College of Medicine, University of Arizona, I50I N Campbell Ave, Tucson, AZ, 85724, USA

Email qchen@email.arizona.edu
Background: Paroxetine is a selective serotonin reuptake inhibitor utilized in the treatment of depression and anxiety disorders. Recent studies have identified paroxetine as a G proteincoupled receptor kinase-2 (GRK2) inhibitor capable of reversing cardiac dysfunction and remodeling in experimental models of acute myocardial infarction (AMI). We determine the clinical importance of paroxetine on cardiac functions in patients having AMI with depression (AMID) in comparison with fluoxetine, an unrelated selective serotonin reuptake inhibitor that does not inhibit GRK2.

Methods: Diagnosis of depression was based on the 17-item Hamilton Depression Scale and Self-rating Depression Scale in AMI patients after hospital admission. AMID patients were randomly assigned to paroxetine or fluoxetine for treatment of depression. Heart rate variability and cardiac function were evaluated. GRK2 protein levels were measured using peripheral lymphocytes and Western blot.

Results: GRK2 expression in AMID patients was significantly higher than that in AMI patients without depression. In AMID patients, GRK2 levels were positively correlated with the 17-item Hamilton Depression Scale and the Self-rating Depression Scale scores, and negatively correlated with heart rate variability. Treatment of AMID patients with paroxetine significantly reduced the expression of GRK2, normalized the autonomic nervous system function, and improved cardiac performance. In contrast, fluoxetine normalized the autonomic nervous system but did not reduce the expression of GRK2 nor improved cardiac performance.

Conclusion: This study suggests that paroxetine is effective for improving cardiac function in patients with AMID and such effect correlates with GRK2 reduction.

Keywords: selective serotonin reuptake inhibitors, heart rate variability, G protein-coupled receptor kinase-2, acute myocardial infarction, depression

\section{Introduction}

Depression is an independent risk factor for poor prognosis in patients suffering from an acute myocardial infarction (AMI). ${ }^{1}$ Approximately $20 \%$ of AMI patients have depressive disorders. ${ }^{2}$ During the past decades, selective serotonin reuptake inhibitors (SSRIs), such as paroxetine and fluoxetine, have been prescribed to patients having AMI with depression (AMID) based on the idea that depression will impose adverse effects on cardiac function. ${ }^{3-6}$ Clinical measures of these SSRIs in cardiac function improvement remains undetermined.

Depression in acute coronary syndromes is often associated with sympathetic hyperactivity and parasympathetic impairment. ${ }^{7,8}$ The imbalance of these autonomic systems is manifested by decreased heart rate variability (HRV). ${ }^{9,10}$ Excessive sympathetic 
activation may contribute to the detrimental effects of depression on cardiac function during or after myocardial infarction (MI). ${ }^{11} \mathrm{HRV}$ analysis is widely used as a noninvasive technique for the determination of autonomic nervous system (ANS) influence on heart rate. ${ }^{12}$ Diminished HRV reflects inadequate cardiac parasympathetic and/or excessive cardiac sympathetic tendencies, ${ }^{9}$ and has been shown to represent an independent predictor of mortality after MI. ${ }^{13}$

Recently, two reports revealed the role of paroxetine as a GRK2 inhibitor and inhibition of GRK2 expression results in improved myocardial contractility in a mouse model of heart failure after AMI., ${ }^{5,6}$ The G protein-coupled receptor kinase $(\mathrm{GRK})$ is a serine/threonine kinase that phosphorylates intracellular domains of activated G-protein coupled receptors, leading to the recruitment of arrestins and attenuation of intracellular $\mathrm{G}$ protein-dependent signaling. Among seven GRKs, GRK2 is important for cardiac function because of its high abundant expression in the heart. ${ }^{14}$ Following a MI, GRK2 expression increases in the myocardium and apparently enhanced GRK2 expression or activity plays a deleterious role in cardiac $\beta$-adrenergic signaling, resulting in desensitization of $\beta$-adrenergic receptor ( $\beta$-AR) or downregulation of $\beta$-AR expression. ${ }^{15,16}$ GRK2 inhibition via genetic knockdown has been shown to effectively improve cardiac function in animal models of heart failure. ${ }^{17-19}$ Iaccarino et al showed that lymphocyte GRK2 levels increased during acute MI and are associated with worse cardiac function. ${ }^{20}$ As a result, lymphocyte GRK2 has been recognized as a potential therapeutic target for the treatment of MI.

Based on these lines of literature, we postulate a measureable clinical benefit of paroxetine via regulating GRK2 expression in AMID patients. In this study, we determined the therapeutic benefits of paroxetine on depression and cardiac function in AMID patients, and measured the correlation of the clinical parameters with GRK2 expression. Fluoxetine, a structurally unrelated SSRI that is effective in managing depression but does not inhibit GRK2 expression, was included as a comparison.

\section{Methods}

\section{Study population}

Patients with confirmed AMI were enrolled in the Coronary Care Unit in Qian Fo Shan Hospital of Shandong University Medical School for inpatient care. These AMI patients had ischemic chest pain for $>30$ minutes but $<24$ hours, persistent ST-segment elevation $\geq 0.1 \mathrm{mV}$, ST-segment depression or T-wave inversion in two adjacent electrocardiography leads, ${ }^{21}$ and significantly elevated blood levels of biomarkers for myocardial injury (creatine kinase-MB and troponin I). Angiography was performed in all patients according to standard procedure. Out of 308 AMI patients recruited, 292 patients finished this 8-week study. Among them, 67 (23\%) were diagnosed with depression based on a score of $\geq 17$ on the 17-item Hamilton Depression Scale (HAMD-17) ${ }^{22}$ and a self-test score using the Self-rating Depression Scale (SDS) ${ }^{23}$ by psychiatrists within 24-48 hours after admission to the Coronary Care Unit. These patients were evaluated over an 8-week treatment period.

This randomized, double-blind study excluded patients who were $>85$ years old, had an infection, allergic disorder, endocrine disease, malignancy, autoimmune disease, rheumatic heart disease, severe liver disease, renal failure, history of drug abuse, or been prescribed with an anti-inflammatory or immunosuppressant drug except aspirin in the past 3 weeks prior to MI. Patients recruited to this study had no history of heart disease prior to AMI, judged by no diagnosis of atrial fibrillation, myocarditis, endocarditis, valvular heart disease, or requirement of an implanted pacemaker. Each patient provided written informed consent, which, along with the study design and protocol, were approved by the Human Subject and Medical Ethics Committee of Qian Fo Shan Hospital of Shandong University. This study was conducted in accordance with the ethical standards established in the 1964 Declaration of Helsinki and its later amendments. ${ }^{24,25}$

\section{Study design}

Medical history of patients, including complete laboratory examination and echocardiographic data, was obtained retroactively through database search. Demographic data, including age, sex, medications, and cardiovascular risk factors, were also collected. Patients were then reassessed using continuous follow-up questionnaire scores and outcome surveys after 8 weeks, including the incidence of postinfarction angina pectoris and recurrent MI. Standard treatments for MI in these patients include aspirin, clopidogrel/ticagrelor, statins, betablockers, calcium blockers, angiotensin-converting enzyme inhibitors, and nitrates. Among 67 AMID patients, 23 were randomly assigned treatment with paroxetine, 23 with fluoxetine, and the rest with no antidepressant treatment for 8 weeks. The dose of paroxetine or fluoxetine was $10 \mathrm{mg} /$ day initially and increased to $20 \mathrm{mg} /$ day within 1 week based on individual patient response.

\section{HRV analysis}

HRV assessments were performed within 24 hours after admission to the Coronary Care Unit. A second HRV recording was performed after 8 weeks of inpatient or outpatient care. 
The frequency domain parameters were obtained by 10-minute recordings with patients in the supine position, as previously described. ${ }^{26}$ Selected standard deviation of NN intervals (SDNN), high frequency (HF, 0.15-0.40 Hz), and low frequency (LF, 0.04-0.15 Hz) measures were used as research indexes. SDNN is considered a measure of global HRV and is a coarse indicator for the status of cardiac autonomic control. ${ }^{27,28}$ HF measures express a phenomenon of respiratory arrhythmia occurring solely due to changes in the vagal input to the heart. LF measures reflect both vagal and sympathetic influences on the heart. ${ }^{27}$ In general, the LF/HF index is considered a measure of the sympathovagal balance in the sinus node.

\section{Western blot analysis for determining GRK2 expression}

GRK2 levels were evaluated at admission and after 8 weeks of treatment for all patients as described previously. ${ }^{29}$ The peripheral blood mononuclear cells were isolated from the whole blood samples by density-gradient centrifugation on Ficoll-Hypaque (Sigma-Aldrich Co., St Louis, $\mathrm{MO}$, USA),$^{30}$ and stored at $-80^{\circ} \mathrm{C}$ until the day of assay. Immunodetection of GRK2 levels was performed by Western blot using polyclonal anti-GRK2 antibodies ${ }^{31}$ (Santa Cruz Biotechnology, Dallas, TX, USA), glyceraldehyde 3-phosphate dehydrogenase antibody (1:2,000; Santa Cruz Biotechnology) was used as an internal loading control. The membranes were incubated with antibody overnight, and then incubated with the corresponding secondary antibody for 1.5 hours. The results were analyzed using Quantity One ImageJ software (National Institute for Health, Bethesda, MD, USA) to quantify the levels of the GRK2 protein.

\section{High performance liquid chromatography (HPLC) analysis of norepinephrine (NE) and epinephrine (EPI) concentrations}

Concentrations of serum EPI and NE were measured by HPLC with electrochemical detection. Peripheral venous blood samples were collected and K2-ethylene-diaminetetraacetic acid (K2-EDTA) was added as anticoagulant. The samples were immediately cooled, centrifuged at $1,500 \times g$ for 5 minutes at $4{ }^{\circ} \mathrm{C}$, and plasma aliquots were stored at $-80^{\circ} \mathrm{C}$, then the concentrations of serum EPI and NE were determined. The aluminum oxide was previously activated with Tris/ EDTA buffer. A total of $50 \mu \mathrm{L}$ internal standard (500 pg dihydroxybenzylamine) and $2 \mathrm{~mL}$ plasma were added in it. After stirring for 10 minutes, it was centrifuged at $800 \times g$ for 1 minute at $4^{\circ} \mathrm{C}$, and washed thrice with Tris/EDTA solution. Aliquots of $50 \mu \mathrm{L}$ were injected into the HPLC system (SHIMADZU Deutschland, Duisburg, Germany).
Electrochemical detection was performed. Single-point calibration curves were calculated.

\section{Statistical analyses}

Data were reported as mean $\pm \mathrm{SD}$. Statistical analyses were performed using the GraphPad Prism 5.0 (GraphPad Software, Inc., La Jolla, CA, USA). Comparison was made using $t$-test for GRK2 levels between AMI and AMID groups. A one-way or two-way analysis of variance or $\chi^{2}$ test was used to compare the different groups as appropriate. The relationships between the depression scores, HRV variables, and GRK2 levels were explored using a Pearson's test. Nonparametric statistics was used in the correlation analysis of HAMD-17 and SDS scores and GRK2 expression in peripheral lymphocytes cells of patients with AMID. Statistical significance was defined as a $P<0.05$.

\section{Results GRK2 protein expression in patients with AMI and AMID}

Table 1 summarizes the baseline characteristics of patients enrolled in this study. There were no significant differences in the ratio of sex, average age, common risk factors for cardiovascular diseases, data from laboratory assays, or drugs from concomitant therapies among the four groups of patients: AMI without depression, AMID without antidepressant, AMID with paroxetine, and AMID with fluoxetine $(P>0.05)$.

\section{The correlations of autonomic nerve function with depressive status and GRK2 expression in AMID patients}

Measures of depression and HRV in AMI patients showed an inverted relationship of all HRV indexes with the HAMD-17 score (Table 2). For SDS, we found correlations with HF and LF/HF. Measurement of GRK2 using lymphocytes from patients' blood showed correlations of GRK2 protein levels with SDNN and LF/HF.

Using nonparametric statistics, we found that GRK2 expression in the peripheral lymphocytes of AMID patients increased with higher HAMD-17 and SDS scores ( $r=0.3746, P=0.0092, \mathrm{n}=67 ; r=0.3471, P=0.0079, \mathrm{n}=67$, Figure 1A and B). Therefore, GRK2 expression in the peripheral lymphocytes of AMID patients positively correlated with the depressive status.

\section{Effects of treatments with paroxetine or fluoxetine in AMID patients}

There were no differences in the protein levels of GRK2 before the treatment among the three AMID groups $(P>0.05$, 
Table I Clinical baseline characteristics of patients

\begin{tabular}{|c|c|c|c|c|}
\hline Variables & AMI $(n=225)$ & AMID-N (n=2I) & AMID-P (n=23) & AMID-F $(n=23)$ \\
\hline Sex (male/female) & $119 / 106$ & $12 / 9$ & $13 / 10$ & $12 / 11$ \\
\hline Age (years) & $60.2 \pm 9.7$ & $62.3 \pm 10.1$ & $63.4 \pm 10.7$ & $61.7 \pm 10.4$ \\
\hline \multicolumn{5}{|l|}{ Risk factors } \\
\hline Smoking, n (\%) & $72(49)$ & $10(50)$ & $12(54)$ & II (48) \\
\hline Family history of IHD, n (\%) & $69(47)$ & $9(45)$ & II (48) & $12(54)$ \\
\hline Obesity, n (\%) & $37(25)$ & $5(27)$ & $5(24)$ & $5(24)$ \\
\hline Diabetes, n (\%) & $43(29)$ & $5(26)$ & $6(29)$ & $6(29)$ \\
\hline Hypertension, n (\%) & $83(56)$ & $12(57)$ & $13(57)$ & $12(54)$ \\
\hline Total cholesterol $(\mathrm{mmol} / \mathrm{L})$ & $4.29 \pm 0.69$ & $4.20 \pm 0.74$ & $4.26 \pm 0.75$ & $4.23 \pm 0.72$ \\
\hline Triglyceride (mmol/L) & $2.23 \pm 2.26$ & $2.36 \pm 2.58$ & $2.33 \pm 2.36$ & $2.35 \pm 2.37$ \\
\hline HDL-cholesterol (mmol/L) & $1.17 \pm 0.28$ & $1.13 \pm 0.29$ & $1.16 \pm 0.24$ & $1.18 \pm 0.25$ \\
\hline LDL-cholesterol (mmol/L) & $3.48 \pm 2.09$ & $3.57 \pm 2.17$ & $3.46 \pm 2.08$ & $3.49 \pm 2.19$ \\
\hline C-reactive protein (mg/L) & $5.25 \pm 7.16$ & $5.15 \pm 8.23$ & $5.26 \pm 7.98$ & $5.17 \pm 7.66$ \\
\hline \multicolumn{5}{|l|}{ Laboratory assay } \\
\hline Myoglobin (ng/mL) & $323.2 \pm 212.4$ & $329.3 \pm 229.1$ & $319 \pm 214.0$ & $324 \pm 221.2$ \\
\hline Creatine kinase-MB (U/L) & $192.3 \pm 144.7$ & $199.2 \pm 163.4$ & $196 \pm 153.1$ & $20 I \pm I 55.3$ \\
\hline Troponin I (ng/mL) & $15.4 \pm 14.39$ & $14.9 \pm 15.03$ & $|4.7 \pm| 4.34$ & $|4.1 \pm| 3.74$ \\
\hline Lymphocyte count $\left(10^{9} / \mathrm{L}\right)$ & $2.2 \pm 1.2$ & $2.1 \pm 0.8$ & $2.3 \pm 1.3$ & $2.2 \pm 1.2$ \\
\hline Number of lesions vessels & $1.4 \pm 0.7$ & $1.6 \pm 0.6$ & $1.5 \pm 0.9$ & $1.5 \pm 0.9$ \\
\hline \multicolumn{5}{|l|}{ Concomitant therapy } \\
\hline Aspirin, n (\%) & $220(98)$ & $21(100)$ & $23(100)$ & $22(97)$ \\
\hline Clopidogrel/ticagrelor, n (\%) & $207(92)$ & $20(9 \mathrm{I})$ & $23(100)$ & $23(100)$ \\
\hline Statins, n (\%) & $225(100)$ & $22(100)$ & $23(100)$ & $23(100)$ \\
\hline Beta-blockers, n (\%) & $175(78)$ & $16(76)$ & $17(74)$ & $18(78)$ \\
\hline ACE inhibitors, $n(\%)$ & $60(27)$ & $6(29)$ & $7(30)$ & $8(34)$ \\
\hline Nitrates, n (\%) & $180(80)$ & $16(76)$ & $18(78)$ & $17(74)$ \\
\hline Calcium blockers, n (\%) & $56(25)$ & $5(24)$ & $6(26)$ & $7(30)$ \\
\hline $\mathrm{PCl}$ + stent, n (\%) & $207(92)$ & $20(95)$ & $21(91)$ & $21(9 I)$ \\
\hline
\end{tabular}

Note: Data was expressed as mean \pm SD.

Abbreviations: ACE, angiotensin-converting enzyme; AMI, acute myocardial infarction; AMID, acute myocardial infarction with depression; F, fluoxetine; HDL, high density lipoprotein; IHD, ischemic heart disease; LDL, low density lipoprotein; N, non-antidepressant; P, paroxetine; PCI, percutaneous coronary intervention; SD, standard deviation.

Figure 2A and B). After concomitant treatments with or without antidepressants for 8 weeks, GRK2 expressions (all $P<0.01$ ) in peripheral lymphocytes were significantly decreased compared to their baseline levels (Figure 2).

Table 2 Correlation analysis of HRV indexes, HAMD-I7 and SDS scores, and GRK2 expression in AMID patients

\begin{tabular}{|c|c|c|c|c|c|c|}
\hline \multirow[t]{2}{*}{ Variables } & \multicolumn{2}{|c|}{ HAMD-I 7} & \multicolumn{2}{|l|}{ SDS } & \multicolumn{2}{|l|}{ GRK2 } \\
\hline & $r$ & $\mathbf{e}$ & $r$ & e & $r$ & ie \\
\hline ) & -0.5653 & & -0.4673 & 0.0531 & -0.5427 & $0.0145^{*}$ \\
\hline & -0 & & & & & \\
\hline (1) & -0.3882 & 0.02 & 0 & 0 & 20 & \\
\hline & -0 & & & & 57 & $7 *$ \\
\hline & 0.5156 & 0.00 & $\mathrm{c}$ & * & 22 & 0.00 \\
\hline $\mathrm{PI}(\mathrm{pg} / \mathrm{mL})$ & 0.4839 & $6 *$ & .5099 & $0.006 I^{* *}$ & 0.5327 & 0.00 \\
\hline \multicolumn{7}{|c|}{$\begin{array}{l}\text { Notes: The Pearson correlation test was used for correlation analysis of the } \\
\text { relationship between } \mathrm{HRV} \text { index and HAMD- } 17 \text { and SDS scores, and GRK2 in AMID } \\
\text { patients. } * P<0.05 \text {. }{ }^{* * P}<0.0 \text {. }\end{array}$} \\
\hline \multicolumn{7}{|c|}{$\begin{array}{l}\text { Abbreviations: AMID, acute myocardial infarction with depression; EPI, } \\
\text { epinephrine; GRK2, G protein-coupled receptor kinase-2; HAMD-17, 17-item } \\
\text { Hamilton Rating Scale for Depression; HF, high frequency; HRV, heart rate variability; }\end{array}$} \\
\hline
\end{tabular}

Notably, GRK2 protein levels showed a greater decrease after paroxetine treatment compared with those of the other two groups (both $P<0.01$ ), while there was no difference in the expression of GRK2 between AMID patients not treated with antidepressant and AMID patients treated with fluoxetine $(P=0.8312)$ (Figure 2A and $\mathrm{B})$.

After treatment of AMID patients with paroxetine or fluoxetine, the HAMD-17 and SDS scores were significantly improved compared with AMID patients without antidepressant $(P<0.01)$, while the benefit effects of paroxetine versus fluoxetine were not different $(P>0.05)$. HRV indexes of the three groups were increased, while EPI and NE were decreased after 8 weeks of treatment (Table 3 ). Interestingly, the improvements of the cardiac function (left ventricular ejection fraction [LVEF]), EPI, NE, HF, LF, and LF/HF in patients following paroxetine treatment were superior to those in patients treated with fluoxetine $(P<0.01)$. The rates of cardiovascular events after AMI, including postinfarction 

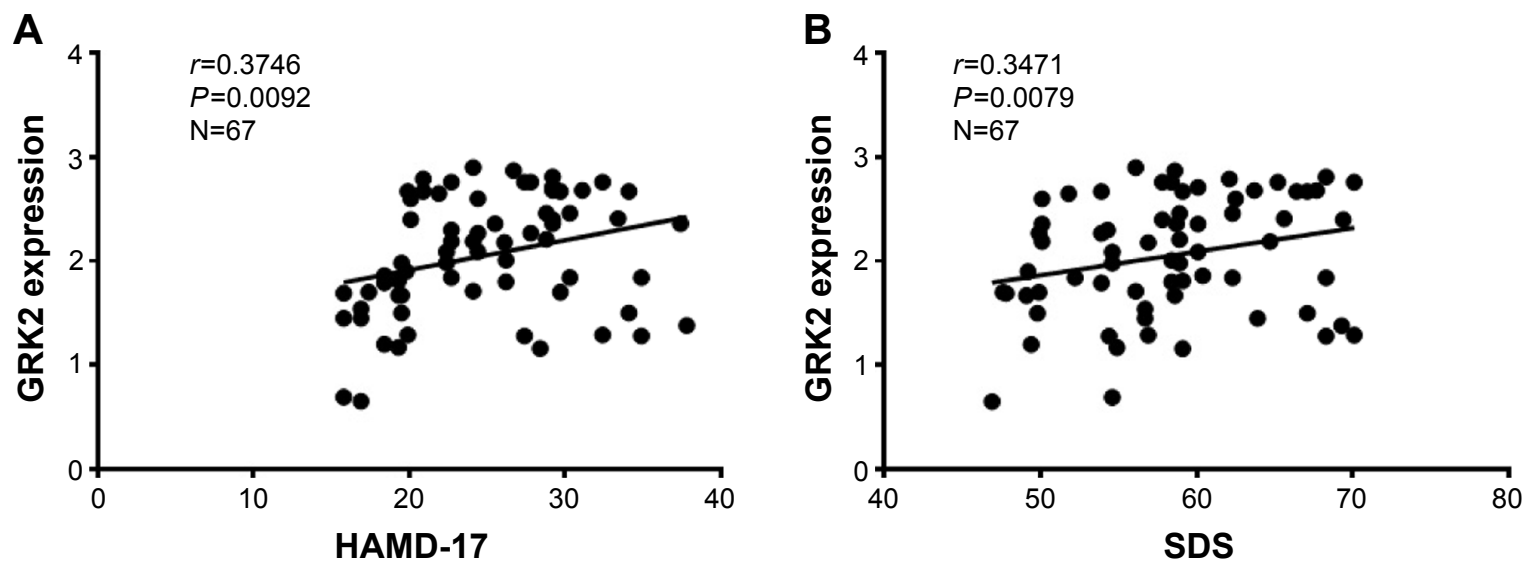

Figure I Correlation analysis of depressive status with GRK2 expression in AMID patients.

Notes: There were positive correlations between HAMD-17 scores and GRK2 expression (A) SDS scores and GRK2 expression (B) in the peripheral lymphocytes of AMID patients.

Abbreviations: AMID, acute myocardial infarction with depression; GRK, G protein-coupled receptor kinases; HAMD-I7, I7-item Hamilton Rating Scale for Depression; SDS, Self-rating Depression Scale.

angina pectoris and recurrent MI were lower in patients receiving antidepressants $(P<0.01)$ (Table 3).

\section{Effects of treatments with antidepressant in AMID and AMI patients}

There were significant increases in the protein levels of GRK2, the HAMD-17, and SDS scores in AMID patients

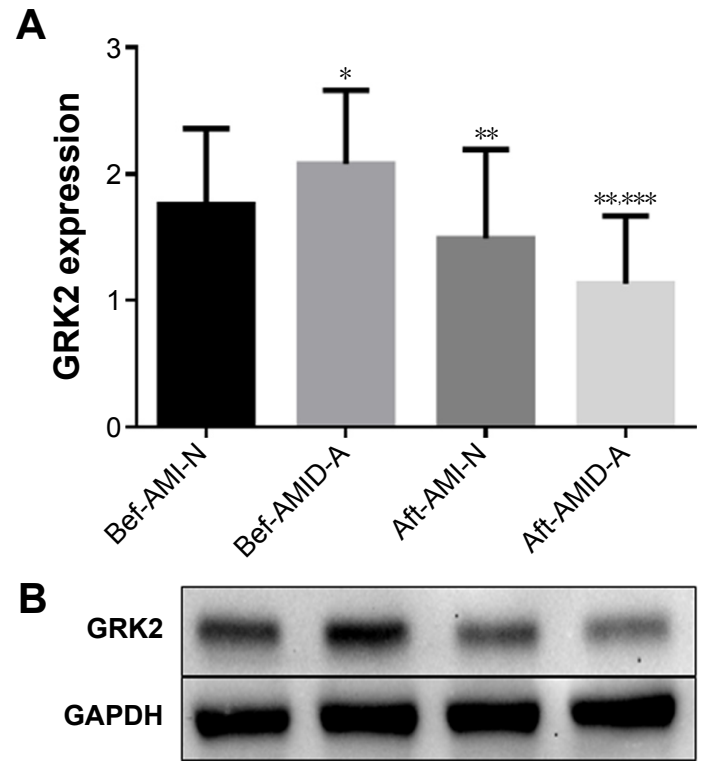

Figure 2 Comparison of (A) and representative data of (B) GRK2 protein expression in the peripheral lymphocytes of AMI patients with or without depression before and after treatment with or without antidepressant.

Notes: Protein expression was normalized against GAPDH. $* P<0.01$ compared with Bef-AMI-N. $* * P<0.0$ I compared with before treatments within groups. $* * * P<0.0$ I compared with Aft-AMI-N.

Abbreviations: Aft, after; AMI-N, acute myocardial infarction without antidepressant; AMID-A, acute myocardial infarction comorbid depression with antidepressant; Bef, before; GAPDH, glyceraldehyde 3-phosphate dehydrogenase; GRK, G protein-coupled receptor kinases; N, non-antidepressant. compared to those of in AMI patients $(P<0.01)$ (Figure 3A and $\mathrm{B}$, Table 4$)$, while there were significant decreases in the HRV indices (SDNN, HF, LF, and LF/HF) in these patients $(P<0.01)$ (Table 4). After concomitant treatments with or without antidepressants for 8 weeks, GRK2 expressions were decreased compared to their baseline levels (Figure 3). Remarkably, GRK2 protein levels showed a greater decrease after antidepressant treatment compared with those of the AMI group $(P<0.01)$ (Figure $3 \mathrm{~A}$ and $\mathrm{B}$ ).

After treatment of AMID patients with antidepressant, the HAMD-17 and SDS scores were significantly improved compared with AMI patients without antidepressant $(P<0.01)$. HRV indices of both the groups were increased, while EPI and NE were decreased after 8 weeks of treatments, but improved more in AMID patients group (Table 4). Significantly, the improvements of the cardiac function (LVEF), EPI, NE, HF, LF, and LF/HF in AMID patients following antidepressant treatment were superior to those in AMI patients $(P<0.01)$ (Table 4).

\section{Effects of treatments with antidepressant in AMID patients of sex before and after treatment with antidepressant}

There were no differences in the protein levels of GRK2, HAMD-17 and SDS scores, HRV indices (SDNN, HF, LF, and LF/HF), and cardiac function (LVEF) before or after treatment in male and female AMID groups $(P>0.05)$ (Table 5). After concomitant treatments with antidepressants for 8 weeks, the above variables were significantly improved compared to their baseline levels $(P<0.01)$ (Table 5). 
Table 3 Comparison of the changes of the variables in AMID patients before and after treatment with or without paroxetine or fluoxetine

\begin{tabular}{|c|c|c|c|c|c|c|}
\hline \multirow[t]{2}{*}{ Variables } & \multicolumn{2}{|c|}{ AMID-N $(n=2 I)$} & \multicolumn{2}{|c|}{ AMID-P $(n=23)$} & \multicolumn{2}{|c|}{ AMID-F $(n=23)$} \\
\hline & Before & After & Before & After & Before & After \\
\hline HAMD-I7 & $27.1 \pm 4.7$ & $26.9 \pm 4.6$ & $27.2 \pm 4.5$ & $11.2 \pm 4.0 * * *$ & $27.4 \pm 4.4$ & $15.9 \pm 4.8^{* * * *}$ \\
\hline SDS & $58.0 \pm 4.4$ & $55.4 \pm 4.8$ & $57.1 \pm 4.3$ & $40.8 \pm 5.7 * * * *$ & $57.5 \pm 4.0$ & $46.9 \pm 4.2^{* * * *}$ \\
\hline LVEF (\%) & $38.1 \pm 4.1$ & $41.4 \pm 4.5$ & $38.0 \pm 5.7$ & $49.8 \pm 6.9 *, * *, * * *$ & $38.2 \pm 4.0$ & $41.7 \pm 5.6 * * *$ \\
\hline FS (\%) & $32.1 \pm 3.2$ & $27.1 \pm 3.9$ & $31.6 \pm 4.0$ & $24.7 \pm 3.7^{*}$ & $31.7 \pm 3.1$ & $28.9 \pm 3.9$ \\
\hline LVDd (mm) & $51.9 \pm 11.3$ & $50.9 \pm 8.6$ & $51.5 \pm 9.2$ & $44.1 \pm 6.3^{*}$ & $51.4 \pm 10.7$ & $47.9 \pm 8.4$ \\
\hline LVPW (mm) & $10.97 \pm 1.0110$ & $34 \pm 1.08$ & $10.08 \pm 1.06$ & $9.46 \pm 1.04$ & $10.16 \pm 1.04$ & $9.46 \pm 1.03$ \\
\hline \multicolumn{7}{|l|}{ HRV } \\
\hline SDNN (ms) & $21 \pm 9$ & $44 \pm 10 *$ & $23 \pm 10$ & $59 \pm 17 * * * *$ & $23 \pm 11$ & $58 \pm 15^{* * * *}$ \\
\hline $\mathrm{HF}\left(\mathrm{ms}^{2}\right)$ & $257 \pm 114$ & $379 \pm 149 *$ & $251 \pm 109$ & $660 \pm 178 *, * *, * * *$ & $255 \pm 106$ & $430 \pm 157^{* * * *}$ \\
\hline $\operatorname{LF}\left(\mathrm{ms}^{2}\right)$ & $328 \pm 173$ & $406 \pm 130 *$ & $358 \pm 176$ & $729 \pm 247 * * * * * * *$ & $346 \pm 152$ & $519 \pm 230 * * * *$ \\
\hline LF/HF (\%) & $48 \pm 32$ & $97 \pm 57 *$ & $42 \pm 46$ & $328 \pm 84 *, * *, * * *$ & $45 \pm 43$ & $189 \pm 68^{* * * *}$ \\
\hline $\mathrm{EPI}(\mathrm{pg} / \mathrm{mL})$ & $115.6 \pm 18.7$ & $76.1 \pm 7.7^{*}$ & $117.4 \pm 19.6$ & $39.2 \pm 5.1 *, * *, * * *$ & $112.5 \pm 17.2$ & $52.4 \pm 5.9 * * * *$ \\
\hline NE (pg/mL) & $928 \pm 367.1$ & $710 \pm 276.3 *$ & $972 \pm 272.4$ & $467 \pm 198.2^{*, * *, * * *}$ & $996 \pm 280.9$ & $589 \pm 253.2 * * * *$ \\
\hline $\mathrm{SBP}(\mathrm{mmHg})$ & $|3| \pm \mid 0.8$ & $124 \pm 9.3$ & $138 \pm 1 \mid .4$ & $115 \pm 7.6$ & $|37 \pm| \mid .8$ & $119 \pm 8.2$ \\
\hline $\mathrm{DBP}(\mathrm{mmHg})$ & $79 \pm 6.9$ & $74 \pm 6.2$ & $84 \pm 7.1$ & $72 \pm 6.0$ & $85 \pm 6.8$ & $73 \pm 6.4$ \\
\hline \multicolumn{7}{|l|}{$\mathrm{CV}$ after $\mathrm{AMI}$} \\
\hline PIAP (\%) & - & $10(47.6)$ & - & $7(30.4)^{* * *}$ & - & $6(26.1)^{* *}$ \\
\hline RMI (\%) & - & $4(19.0)$ & - & $3(13.0) * *$ & - & $3(13.0) * *$ \\
\hline
\end{tabular}

Notes: Data was expressed as mean \pm SD. $* P<0.01$ compared with before treatment within groups. $* * P<0.01$ compared with after treatments of AMID $+N$. $* * * P<0.01$ compared with after treatments of fluoxetine.

Abbreviations: AMI, acute myocardial infarction; AMID, acute myocardial infarction with depression; CV, cardiovascular events; DBP, diastolic blood pressure; EPI, epinephrine; F, fluoxetine; FS, shortening fraction; HAMD- 17, I7-item Hamilton Depression Scale for Depression; HF, high frequency; HRV, heart rate variability; LF, low frequency; LF/HF, LF-to-HF ratio; LVDd, LV inner diameter during diastole; LVEF, left ventricular ejection fraction; LVPW, LV posterior wall thickness in diastole; N, nonantidepressant; NE, norepinephrine; P, paroxetine; PIAP, postinfarction angina pectoris; RMI, recurrent myocardial infarction after infarction; SBP, systolic blood pressure; SD, standard deviation; SDS, Self-rating Depression Scale; SDNN, standard deviation of all normal beats.

A

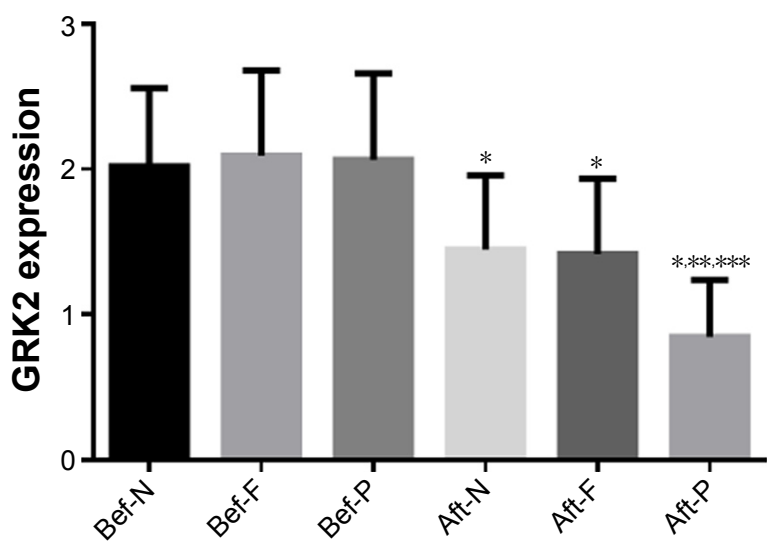

B

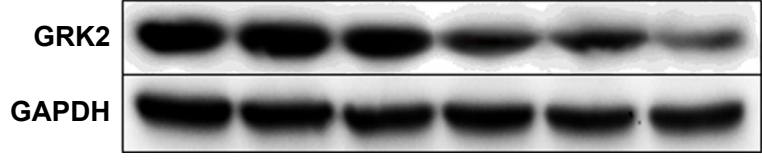

Figure 3 Comparison of $(\mathbf{A})$ and representative data of $(\mathbf{B})$ GRK2 protein expression in AMID patients before and after treatment with or without antidepressants.

Notes: Protein expression was normalized against GAPDH. $* P<0.01$ compared with before treatments within groups. ${ }^{*} P P<0.01$ compared with after treatment of non-antidepressant. $* * * P<0.0$ I compared with after treatment of fluoxetine.

Abbreviations: Aft, after; AMID, acute myocardial infarction with depression; Bef, before; F, fluoxetine; GAPDH, glyceraldehyde 3-phosphate dehydrogenase; GRK, G protein-coupled receptor kinases; N, non-antidepressant; P, paroxetine.

\section{Discussion}

This study is the first to reveal the effect of depression on GRK2 expression and cardiac function in AMI patients. Compared with AMI patients without depression, AMID patients exhibited higher levels of EPI and NE, upper scores of SDS and HAMD-17, greater expression of GRK2, and lower HRV indices. In AMID patients, both "subjective" HAMD-17 and "objective" SDS measures were used at most assessment time points. ${ }^{32}$ The HAMD is often considered as the "gold standard" for evaluating depression severity or treatment efficacy in antidepressant clinical trials. ${ }^{33} \mathrm{We}$ found that higher levels of GRK2 expression correlates with worse scores of HAMD-17 and SDS, indices of HRV, and higher levels of EPI and NE in AMID patients. Our findings indicate that depression leads to hyperactivity of the sympathetic system, suppressed vagal activity, and increased GRK2 expression in AMI patients.

Depression promotes ANS hyperactivity. Chronic deficiency of neurotransmitters, such as 5-hydroxy tryptamine in the synaptic cleft of neurons in patients with depression blocks the central sympathetic inhibitory signals, leading to persistent sympathetic system activation. ${ }^{34} \mathrm{HRV}$, an indirect 
Table 4 Comparison of the changes of the variables in AMI patients with or without depression before and after treatment with or without antidepressant

\begin{tabular}{|c|c|c|c|c|}
\hline \multirow[t]{2}{*}{ Variables } & \multicolumn{2}{|c|}{ AMI-N (n=225) } & \multicolumn{2}{|c|}{ AMID-A $(n=46)$} \\
\hline & Before & After & Before & After \\
\hline HAMD-I7 & $7.1 \pm 3.6$ & $7.6 \pm 4.5$ & $27.3 \pm 4.5^{* *}$ & $13.5 \pm 4.9 * * * * *$ \\
\hline SDS & $38.7 \pm 7.9$ & $38.4 \pm 7.8$ & $57.3 \pm 4.6 * *$ & $43.7 \pm 5.5^{* * * * *}$ \\
\hline LVEF (\%) & $38.3 \pm 4.2$ & $42.3 \pm 5.8^{*}$ & $38.1 \pm 5.8$ & $47.9 \pm 7.0 * * * *$ \\
\hline \multicolumn{5}{|l|}{ HRV } \\
\hline SDNN (ms) & $32 \pm 10$ & $49 \pm 11 *$ & $23 \pm 11 * *$ & $59 \pm 18 * * * * *$ \\
\hline $\mathrm{HF}\left(\mathrm{ms}^{2}\right)$ & $379 \pm 136$ & $489 \pm 153^{*}$ & $253 \pm 110 * *$ & $587 \pm 183^{* * * * * *}$ \\
\hline $\operatorname{LF}\left(\mathrm{ms}^{2}\right)$ & $428 \pm 173$ & $576 \pm 130 *$ & $350 \pm 177 * *$ & $643 \pm 249 * * * * *$ \\
\hline LF/HF (\%) & $69 \pm 36$ & $104 \pm 87^{*}$ & $44 \pm 46 * *$ & $304 \pm 94^{*} * * * *$ \\
\hline EPI (pg/mL) & $89.8 \pm 13.2$ & $54.2 \pm 8.9 *$ & $114.8 \pm 19.7^{* *}$ & $46.1 \pm 5.9 * * * * *$ \\
\hline $\mathrm{NE}(\mathrm{pg} / \mathrm{mL})$ & $838.2 \pm 154.7$ & $692.5 \pm 108.9 *$ & $983.1 \pm 281.9 * *$ & $510.6 \pm 176.3^{* * * * *}$ \\
\hline $\mathrm{SBP}(\mathrm{mmHg})$ & $134 \pm 14.8$ & $127 \pm 10.3$ & $138 \pm 11.9$ & $117 \pm 8.3^{*}$ \\
\hline $\mathrm{DBP}(\mathrm{mmHg})$ & $8 I \pm 7.4$ & $75 \pm 6.7$ & $85 \pm 7.2$ & $72 \pm 6.5$ \\
\hline
\end{tabular}

Notes: Data was expressed as mean \pm SD. $* P<0.01$ compared with before treatment within groups. $* * P<0.01$ compared with before treatments of AMI. $* * * P<0.01$ compared with after treatments of AMI.

Abbreviations: A, antidepressant; AMI, acute myocardial infarction; AMID, acute myocardial infarction with depression; DBP, Diastolic blood pressure; EPI, epinephrine; HAMD- 17, I7-item Hamilton Depression Scale for Depression; HF, high frequency; HRV, heart rate variability; LF, low frequency; LF/HF, LF-to-HF ratio; LVEF, left ventricular ejection fraction; N, non-antidepressant; NE, norepinephrine; SBP, systolic blood pressure; SD, standard deviation; SDS, Self-rating Depression Scale; SDNN, standard deviation of all normal beats.

quantitative measure of the ANS, decreases in patients with depression and the degree of decrease reflects the severity of depression. ${ }^{35,36}$ In frequency domain parameters, HF expresses a phenomenon of respiratory arrhythmia that occurs solely due to changes in the vagal input to the heart,

Table 5 Comparison of the changes of the variables in AMID patients of gender before and after treatment with antidepressant

\begin{tabular}{|c|c|c|c|c|}
\hline \multirow[t]{2}{*}{ Variables } & \multicolumn{2}{|c|}{ AMID + male $(n=25)$} & \multicolumn{2}{|c|}{ AMID + female $(n=2 I)$} \\
\hline & Before & After & Before & After \\
\hline HAMD-I7 & $27.1 \pm 4.1$ & $13.7 \pm 4.5^{*}$ & $27.8 \pm 4.2$ & $13.3 \pm 4.6^{*}$ \\
\hline SDS & $56.2 \pm 4.4$ & $25.8 \pm 5.8^{*}$ & $58.5 \pm 4.9$ & $26.7 \pm 6.0 *$ \\
\hline LVEF (\%) & $38.3 \pm 5.6$ & $41.5 \pm 4.9$ & $38.6 \pm 6.0$ & $49.5 \pm 6.8^{*}$ \\
\hline \multicolumn{5}{|l|}{ HRV } \\
\hline SDNN (ms) & $23 \pm 10$ & $57 \pm 18 *$ & $23 \pm 11$ & $61 \pm 19 *$ \\
\hline $\mathrm{HF}\left(\mathrm{ms}^{2}\right)$ & $250 \pm 108$ & $549 \pm 176^{*}$ & $257 \pm 109$ & $592 \pm 183^{*}$ \\
\hline $\operatorname{LF}\left(\mathrm{ms}^{2}\right)$ & $358 \pm 176$ & $626 \pm 246^{*}$ & $346 \pm 172$ & $658 \pm 248^{*}$ \\
\hline LF/HF (\%) & $44 \pm 48$ & $293 \pm 90 *$ & $42 \pm 44$ & $316 \pm 97^{*}$ \\
\hline GRK2 & $2.04 \pm 0.57$ & I. $12 \pm 0.54^{*}$ & $2.10 \pm 0.58$ & I. $14 \pm 0.54 *$ \\
\hline $\mathrm{EPI}(\mathrm{pg} / \mathrm{mL})$ & $118.1 \pm 19.6$ & $48.8 \pm 5.6 *$ & $116.3 \pm 19.7$ & $44.6 \pm 5.8^{*}$ \\
\hline NE (pg/mL) & $972.8 \pm 272.8$ & $518.2 \pm 177.4^{*}$ & $992.3 \pm 279.8$ & $502.4 \pm 174.9 *$ \\
\hline $\mathrm{SBP}(\mathrm{mmHg})$ & $|36 \pm| \mid .5$ & $116 \pm 8.1$ & $|4| \pm|| .7$ & $128 \pm 8.2 *$ \\
\hline $\mathrm{DBP}(\mathrm{mmHg})$ & $87 \pm 7.1$ & $71 \pm 6.3$ & $82 \pm 7.1$ & $74 \pm 6.4$ \\
\hline
\end{tabular}

Notes: Data was expressed as mean $\pm S D$. $* P<0.01$ compared with before treatment within groups.

Abbreviations: AMID, acute myocardial infarction with depression; DBP, diastolic blood pressure; EPI, epinephrine; GRK2, G protein-coupled receptor kinase-2; HAMD-17, 17-item Hamilton Depression Scale for Depression; HF, high frequency; HRV, heart rate variability; LF, low frequency; LF/HF, LF-to-HF ratio; LVEF, left ventricular ejection fraction; NE, norepinephrine; SBP, systolic blood pressure; SD, standard deviation; SDNN, standard deviation of all normal beats; SDS, Self-rating Depression Scale. whereas LF reflects both vagal and sympathetic influences on the heart. ${ }^{27}$ The LF/HF index is considered a measure of the sympathovagal balance in the sinus node. In agreement with previous research, ${ }^{12,35,37}$ we identified that $\mathrm{LF}$ and LF/HF indexes were markedly decreased in patients with depression. This type of ANS hyperactivity was also documented in acute coronary syndromes with concomitant depression. ${ }^{7,8}$ The adverse effect of depression on cardiac function in a MI patient may be related to sympathetic activation. ${ }^{32}$

GRK2 might be increased due to $\beta$-AR activation by catecholamines, which result from sympathetic hyperactivation. An increasing body of evidence suggests that GRK2 participates cardiac remodeling, which involves neurohormonal mechanisms. ${ }^{38-41}$ GRK2 negatively regulates nitric oxide synthase activity, ${ }^{39}$ glucose metabolism, ${ }^{37,40}$ and mitochondrial function, ${ }^{41}$ in the myocardium. GRK2 overexpression in the heart of mice weakened isoproterenol-stimulated contractility, reduced cyclic adenosine monophosphate levels, and impaired cardiac function. ${ }^{42}$ In a mouse model of MI, adrenal-targeted GRK2 knockout diminished sympathetic activation, which attenuated heart failure progression and improved cardiac function after MI. ${ }^{38}$ Therefore, GRK2 inhibition preserves protective signal via a novel sympatholytic pathway, resulting in an improved cardiac contractility and resistance against HF progression after MI.

SSRI antidepressants, such as paroxetine and fluoxetine, have been used for the treatment of depression, including ischemic heart disease patients with depression. ${ }^{43}$ Our study 
found that treatment with antidepressants increased HRV indices. Interestingly, although paroxetine and fluoxetine had comparable antidepression effects, three HRV indices (HF, LF, and LF/HF) of the paroxetine group were higher than those of the fluoxetine group. These results suggest that antidepressants could be beneficial for patients suffering from ANS imbalance and that paroxetine may have a direct mechanism in addition to SSR inhibition. Thal et $a 1^{5}$ reported an interesting discovery that paroxetine has a higher selectivity for inhibition of GRK2 activity than other GRKs, and that paroxetine potentiated cardiac contraction while fluoxetine had no effects. A recent report showed that paroxetine considerably improved left ventricular function and structure in a mouse model forMI. ${ }^{6}$ This effect was due to its ability to inhibit GRK2 instead of SSR. This is consistent with our findings from clinical studies as reported here.

In this study, we found that AMID patients showed increased GRK2 expression. Notably, after 2 months of treatment, NE, EPI, and the GRK2 levels were lower in patients treated with paroxetine than those treated with fluoxetine or no antidepressant. This suggests better cardiac function in the paroxetine group as a result of the inhibition of deleterious effects of GRK2 to the heart, both directly or indirectly. But experiments in laboratory animals ${ }^{6}$ showed that fluoxetine did not have much influence in GRK2 expression, the reason may be the differences of dose and duration of treatment; above all, we compared AMI with AMID patients, and not just AMI patients only. While in male and female patients, there were no sex differences in the effects of antidepressant therapy. As mentioned earlier, elevated activity of GRK2 in the adrenal gland contributes to sympathetic overactivity and enhanced circulation of catecholamines during chronic heart failure. ${ }^{38}$ It was reported that paroxetine inhibited the activity of GRK2 with a relatively high potency, ${ }^{5}$ which suggests that, in our study, the extra effects of paroxetine might be at least partially due to its direct inhibition on GRK2. Indeed, we found that LVEF, fractional shortening, and left ventricular dysfunction were markedly improved in AMID patients treated with paroxetine. In contrast, fluoxetine treatment and no antidepressant treatment caused insignificant change in cardiac function.

Despite the fact that we cannot draw a firm conclusion concerning the superiority of paroxetine over fluoxetine for AMID patients, observations made during this study can still shed some light on the hypothesis that paroxetine correlates with improved heart function recovery in AMID patients.

\section{Conclusion}

In conclusion, the data from this study suggest the benefit of using paroxetine to improve cardiac function in AMID patients and such effect associates with GRK2 reduction.

\section{Acknowledgment}

This work was supported by the Tackle Key Problems in Science and Technology Program of Shandong Province (grant no 2012YD18051).

\section{Disclosure}

The authors report no conflicts of interest in this work.

\section{References}

1. Lichtman JH, Froelicher ES, Blumenthal JA, et al. Depression as a risk factor for poor prognosis among patients with acute coronary syndrome: systematic review and recommendations: a scientific statement from the American Heart Association. Circulation. 2014;129(12): $1350-1369$.

2. Smolderen KG, Spertus JA, Reid KJ, et al. The association of cognitive and somatic depressive symptoms with depression recognition and outcomes after myocardial infarction. Circ Cardiovasc Qual Outcomes. 2009;2(4):328-337.

3. Roose SP, Laghrissi-Thode F, Kennedy JS, et al. Comparison of paroxetine and nortriptyline in depressed patients with ischemic heart disease. JAMA. 1998;279(4):287-291.

4. Arroll B, Macgillivray S, Ogston S, et al. Efficacy and tolerability of tricyclic antidepressants and SSRIs compared with placebo for treatment of depression in primary care: a meta-analysis. Ann Fam Med. 2005; 3(5):449-456.

5. Thal DM, Homan KT, Chen J, et al. Paroxetine is a direct inhibitor of g protein-coupled receptor kinase 2 and increases myocardial contractility. ACS Chem Biol. 2012;7(11):1830-1839.

6. Schumacher SM, Gao E, Zhu W, et al. Paroxetine-mediated GRK2 inhibition reverses cardiac dysfunction and remodeling after myocardial infarction. Sci Transl Med. 2015;7(277):277ra231.

7. Guinjoan SM, de Guevara MS, Correa C, et al. Cardiac parasympathetic dysfunction related to depression in older adults with acute coronary syndromes. J Psychosom Res. 2004;56(1):83-88.

8. Carney RM, Freedland KE, Veith RC. Depression, the autonomic nervous system, and coronary heart disease. Psychosom Med. 2005;67(Suppl 1): S29-S33.

9. Carney RM, Freedland KE, Stein PK, Skala JA, Hoffman P, Jaffe AS. Change in heart rate and heart rate variability during treatment for depression in patients with coronary heart disease. Psychosom Medicine. 2000; 62(5):639-647.

10. Harris PR, Sommargren CE, Stein PK, Fung GL, Drew BJ. Heart rate variability measurement and clinical depression in acute coronary syndrome patients: narrative review of recent literature. Neuropsychiatr Dis Treat. 2014;10:1335-1347.

11. Shi S, Liang J, Liu T, et al. Depression increases sympathetic activity and exacerbates myocardial remodeling after myocardial infarction: evidence from an animal experiment. PloS One. 2014;9(7):e101734.

12. Carney RM, Blumenthal JA, Freedland KE, et al. Low heart rate variability and the effect of depression on post-myocardial infarction mortality. Arch Intern Med. 2005;165(13):1486-1491.

13. Amadi A, Ponikowski P, Coats AJ. Role of catecholamines and sympathetic activation as a risk factor for coronary artery disease. J Cardiovasc Risk. 1995;2(3):222-228.

14. Zhu W, Petrashevskaya N, Ren S, et al. Gi-biased beta2AR signaling links GRK2 upregulation to heart failure. Circ Res. 2012;110(2):265-274. 
15. Seibold A, Williams B, Huang ZF, et al. Localization of the sites mediating desensitization of the beta(2)-adrenergic receptor by the GRK pathway. Mol Pharmacol. 2000;58(5):1162-1173.

16. Rengo G, Perrone-Filardi P, Femminella GD, et al. Targeting the beta-adrenergic receptor system through G-protein-coupled receptor kinase 2: a new paradigm for therapy and prognostic evaluation in heart failure: from bench to bedside. Circ Heart Fail. 2012;5(3):385-391.

17. Lymperopoulos A, Rengo G, Koch WJ. GRK2 inhibition in heart failure: something old, something new. Curr Pharm Des. 2012;18(2): 186-191.

18. Cannavo A, Liccardo D, Koch WJ. Targeting cardiac beta-adrenergic signaling via GRK2 inhibition for heart failure therapy. Front Physiol. 2013;4:264.

19. Salazar NC, Vallejos X, Siryk A, et al. GRK2 blockade with betaARKct is essential for cardiac beta2-adrenergic receptor signaling towards increased contractility. Cell Commun Signal. 2013;11:64.

20. Iaccarino G, Barbato E, Cipolletta E, et al. Elevated myocardial and lymphocyte GRK2 expression and activity in human heart failure. Eur Heart J. 2005;26(17):1752-1758.

21. Almendro-Delia M, Gonzalez-Torres L, Garcia-Alcantara A, et al. Prognostic impact of clopidogrel pretreatment in patients with acute coronary syndrome managed invasively. Am J Cardiol. 2015;115(8): s1019-s1026.

22. Hamilton M. A rating scale for depression. J Neurol, Neurosurg, Psychiatry. 1960;23:56-62.

23. Zung WW, Richards CB, Short MJ. Self-rating depression scale in an outpatient clinic. Further validation of the SDS. Arch Gen Psychiatry. 1965;13(6):508-515.

24. World Medical Association General A. World Medical Association Declaration of Helsinki: ethical principles for medical research involving human subjects. Int J Bioethics. 2004;15(1):124-129.

25. World Medical A. World Medical Association Declaration of Helsinki: ethical principles for medical research involving human subjects. JAMA. 2013;310(20):2191-2194.

26. Liu CC, Kuo TB, Yang CC. Effects of estrogen on gender-related autonomic differences in humans. Am J Physiol. 2003;285(5): H2188-H2193.

27. Singh N, Mironov D, Armstrong PW, Ross AM, Langer A. Heart rate variability assessment early after acute myocardial infarction. Pathophysiological and prognostic correlates. GUSTO ECG Substudy Investigators. Global utilization of streptokinase and TPA for occluded arteries. Circulation. 1996;93(7):1388-1395.

28. Vigo DE, Nicola Siri L, Ladron De Guevara MS, et al. Relation of depression to heart rate nonlinear dynamics in patients $>$ or $=60$ years of age with recent unstable angina pectoris or acute myocardial infarction. Am J Cardiol. 2004;93(6):756-760.

29. Santulli G, Campanile A, Spinelli L, et al. G protein-coupled receptor kinase 2 in patients with acute myocardial infarction. Am J Cardiol. 2011; 107(8):1125-1130.

30. Boyum A. Isolation of mononuclear cells and granulocytes from human blood. Isolation of mononuclear cells by one centrifugation, and of granulocytes by combining centrifugation and sedimentation at $1 \mathrm{~g}$. Scand J Clin Lab Invest Suppl. 1968;97:77-89.
31. Hata JA, Williams ML, Schroder JN, et al. Lymphocyte levels of GRK2 (betaARK1) mirror changes in the LVAD-supported failing human heart: lower GRK2 associated with improved beta-adrenergic signaling after mechanical unloading. J Card Fail. 2006;12(5):360-368.

32. Qu SS, Huang Y, Zhang ZJ, et al. A 6-week randomized controlled trial with 4-week follow-up of acupuncture combined with paroxetine in patients with major depressive disorder. J Psychiatr Res. 2013;47(6): 726-732.

33. Zimmerman M, McGlinchey JB, Posternak MA, Friedman M, Boerescu D, Attiullah N. Differences between minimally depressed patients who do and do not consider themselves to be in remission. J Clin Psychiatry. 2005;66(9):1134-1138.

34. Brown AD, Barton DA, Lambert GW. Cardiovascular abnormalities in patients with major depressive disorder: autonomic mechanisms and implications for treatment. CNS Drugs. 2009;23(7):583-602.

35. Blood JD, Wu J, Chaplin TM, et al. The variable heart: High frequency and very low frequency correlates of depressive symptoms in children and adolescents. J Affect Disord. 2015;186:119-126.

36. Frasure-Smith N, Lesperance F, Irwin MR, Talajic M, Pollock BG. The relationships among heart rate variability, inflammatory markers and depression in coronary heart disease patients. Brain Behav Immun. 2009; 23(8):1140-1147.

37. Woodall MC, Ciccarelli M, Woodall BP, Koch WJ. G protein-coupled receptor kinase 2: a link between myocardial contractile function and cardiac metabolism. Circ Res. 2014;114(10):1661-1670.

38. Lymperopoulos A, Rengo G, Gao E, Ebert SN, Dorn GW 2nd, Koch WJ. Reduction of sympathetic activity via adrenal-targeted GRK2 gene deletion attenuates heart failure progression and improves cardiac function after myocardial infarction. J Biol Chem. 2010; 285(21):16378-16386.

39. Huang ZM, Gao E, Fonseca FV, et al. Convergence of G protein-coupled receptor and S-nitrosylation signaling determines the outcome to cardiac ischemic injury. Sci Signal. 2013;6(299):ra95.

40. Ciccarelli M, Chuprun JK, Rengo G, et al. G protein-coupled receptor kinase 2 activity impairs cardiac glucose uptake and promotes insulin resistance after myocardial ischemia. Circulation. 2011;123(18): 1953-1962.

41. Chen M, Sato PY, Chuprun JK, et al. Prodeath signaling of G proteincoupled receptor kinase 2 in cardiac myocytes after ischemic stress occurs via extracellular signal-regulated kinase-dependent heat shock protein 90-mediated mitochondrial targeting. Circ Res. 2013;112(8): 1121-1134.

42. Koch WJ, Rockman HA, Samama P, et al. Cardiac function in mice overexpressing the beta-adrenergic receptor kinase or a beta ARK inhibitor. Science. 1995;268(5215):1350-1353.

43. Jiang W, Davidson JR. Antidepressant therapy in patients with ischemic heart disease. Am Heart J. 2005;150(5):871-881.
Neuropsychiatric Disease and Treatment

\section{Publish your work in this journal}

Neuropsychiatric Disease and Treatment is an international, peerreviewed journal of clinical therapeutics and pharmacology focusing on concise rapid reporting of clinical or pre-clinical studies on a range of neuropsychiatric and neurological disorders. This journa is indexed on PubMed Central, the 'PsycINFO' database and CAS,

\section{Dovepress}

and is the official journal of The International Neuropsychiatric Association (INA). The manuscript management system is completely online and includes a very quick and fair peer-review system, which is all easy to use. Visit http://www.dovepress.com/testimonials.php to read real quotes from published authors. 